\title{
Um Modelo Conceitual de Probabilidade para Determinação da Vulnerabilidade Populacional ao Clima
}

\author{
Bruce Kelly da Nobrega Silva ${ }^{1}$ (D, Paulo Sergio Lucio ${ }^{1}$, Fabrício Daniel dos Santos Silva ${ }^{2}$, \\ Madson Tavares Silva ${ }^{3}$, Rafaela Lisboa Costa ${ }^{2}$, Edivaldo Afonso de Oliveira Serrão ${ }^{3}$, \\ Vicente de Paulo Rodrigues da Silva ${ }^{3}$, Rodrigo Lins da Rocha Júnior ${ }^{2}$ \\ ${ }^{1}$ Programa de Pós-Graduação em Ciências Climáticas, \\ Universidade Federal do Rio Grande do Norte, Natal, RN, Brasil. \\ ${ }^{2}$ Instituto de Ciências Atmosféricas, Universidade Federal de Alagoas, Maceió, AL, Brasil. \\ ${ }^{3}$ Departamento de Ciências Atmosféricas, Universidade Federal de Campina Grande, \\ Campina Grande, PB, Brasil.
}

Recebido em: 21 de Julho de 2020 - Aceito em: 16 de Agosto de 2020

\begin{abstract}
Resumo
A avaliação da vulnerabilidade da população é uma análise dos impactos esperados, modelagem de risco, exposição, sensibilidade e falta de capacidade de adaptação de uma região ou de um setor específico aos efeitos de eventos climáticos extremos. A vulnerabilidade engloba uma variedade de conceitos incluindo sensibilidade ou suscetibilidade a danos e falta de capacidade para lidar e adaptar. Uma forma de analisar estes aspectos tão diferenciados é através de um processo estocástico, como a probabilidade condicional. Neste artigo, apresenta-se um modelo conceitual para avaliar a vulnerabilidade da população ao clima, levando em conta uma das regiões susceptíveis brasileiras, a Nordeste. Os resultados mostram que o indicador proposto IVPopS apresenta a região central semiárida mais vulnerável, além de uma avaliação detalhada em cada componente do indicador. Áreas produtivas como Petrolina-PE estão com índices elevados de risco $(0,604)$, exposição $(0.863)$, sensibilidade $(0,910)$, enquanto a incapacidade de adaptação está com índices baixos $(0,002)$.
\end{abstract}

Palavras-chave: risco, sensibilidade climática, seca, análise multivariada, população.

\section{A Conceptual Model of Probability for Determining Population Vulnerability to Climate}

\begin{abstract}
The population's vulnerability assessment is an analysis of the expected impacts, risk modeling, exposure, sensitivity and lack of adaptability of a specific region or sector to the effects of extreme weather events. The vulnerability encompasses a variety of concepts including sensitivity or susceptibility to harm and a lack of ability to cope and adapt. One way to analyze these very different aspects is through a stochastic process, such as conditional probability. In this article, a conceptual model is presented to assess the population's vulnerability to the climate, taking into account one of the susceptible regions of Brazil, the Northeast. The results show that the proposed indicator IVPopS presents the most vulnerable central semi-arid region, in addition to a detailed assessment in each component of the indicator. Production areas such as Petrolina-PE have high levels of risk $(0,604)$, exposure $(0,863)$, sensitivity $(0,910)$, while the inability to adapt is low (0.002).
\end{abstract}

Keywords: risk, climate sensitivity, drought, multivariate analysis, population.

\section{Introdução}

O quinto relatório de análises (AR5-Sigla do inglês, Fifth Assessment Report) do Intergovernmental Panel on
Climate Change (IPCC, 2014) afirma que vulnerabilidade é uma propensão ou predisposição de um sistema ser afetado de forma adversa, cujo contexto engloba uma varie-

Autor de correspondência: Bruce Kelly, brucekellys@hotmail.com. 
dade de conceitos e elementos, incluindo sensibilidade ou susceptibilidade, perdas, danos e a falta de capacidade para lidar com este evento estressor e adaptar-se.

Determinar esses termos (ou compartimentos) é necessário para definir os rumos das pesquisas nesta área, pois os riscos socioambientais associados as interações climáticas não são apenas circunstâncias geradas externamente pelos sistemas climáticos, mas resultados de interações complexas entre sociedades e ecossistemas (Obermaier e Rosa, 2013). Embora a exposição a um perigo possa ser a mesma em diferentes grupos sociais, os impactos são determinados de forma variável devido à incapacidade de mitigação dos efeitos (Silva e Lucio, 2014a).

Historicamente o Nordeste Brasileiro (NEB) enfrenta muitas dificuldades para mitigar os efeitos causados pelas longas estiagens, ou secas na região. Diversos estudos analisam a padrão de temperatura na superfície do mar, como o proposto por Franchito et al. (2015), onde sugere que a atuação do fenômeno atmosférico/oceano denominado de El Niño Oscilação Sul (ENOS), que refere-se às situações nas quais o oceano Pacífico Equatorial está mais quente (El Niño) ou mais frio (La Niña) do que a média normal histórica. Essa alteração na temperatura do oceano Pacífico Equatorial acarreta efeitos globais na temperatura e precipitação, na faixa equatorial a convecção se desloca para o leste, alterando o posicionamento da Célula de Walker.

Segundo Nóbrega et al. (2016), quando se configura o fenômeno El Niño ocorre o deslocamento da Célula de Walker para o Pacífico Leste devido ao aquecimento anômalo da superfície do mar, favorecendo subsidência de ar sobre a bacia centro-oeste e sobre o Atlântico Equatorial, incluindo a Amazônia Oriental e o Nordeste do Brasil, o que explicaria episódios de seca nessa região.

Outro fenômeno oceano/atmosférico que está associado a mudança anômala na temperatura da superfície da água do mar no oceano Atlântico Tropical é conhecida como Dipolo do Atlântico, caracterizado pelo gradiente de temperatura entre as águas do Atlântico Tropical Norte estão mais quentes e as águas do Atlântico Equatorial e Tropical Sul estão mais frias existem movimentos descendentes transportando ar frio e seco dos altos níveis da atmosfera sobre a região setentrional, central e sertão do Nordeste inibindo a formação de nuvens e diminuindo a precipitação (Fase Positiva do Dipolo), podendo causar secas (Nóbrega et al., 2016).

A seca como desastre natural afeta milhares de pessoas no NEB. Fatores associados a este tipo de evento relacionam-se a impactos socioeconômicos como queda da produção agrícola (Guiqin et al., 2012), principalmente no que tange aos pequenos produtores (Kienberger et al., 2009; Silva e Lucio, 2014), ou diminuição da produção energética e abastecimento de água devido à escassez dos reservatórios (Brooks et al., 2005), além de impactos na saúde como surtos endêmicos e problemas associados à pouca qualidade da água como endoparasitas (Haines et al., 2006).

Nos últimos anos os estudos concentraram-se na análise de risco da população exposta, na avaliação dos efeitos e em como mitigar os danos causados por eventos climáticos extremos. A interação dos elementos desse conjunto se traduz na vulnerabilidade populacional aos efeitos do clima, com a criação de diversos índices de vulnerabilidade, como o índice de vulnerabilidade social (SCVI - Sigla do inglês, Social-Climatic Vulnerability Index) proposto por Torres et al. (2012), ou que são indicadores da exposição ao perigo de eventos climáticos extremos, por exemplo, o indicador de risco/exposição à seca $I s$, desenvolvido por (Silva e Lucio, 2014a).

$\mathrm{O}$ crescimento dos aglomerados urbanos tornou as relações entre ambiente natural e humanidade mais complexas, onde os processos de ocupação se deram de forma vertiginosa e intensa causando a queda das condições ambientais nas múltiplas esferas acarretando uma desvalorização econômica e empobrecimento de certos grupos sociais (Fialho e Souza, 2007). Hummel et al. (2016) destaca a importância de medir a vulnerabilidade social em níveis subnacionais devido a heterogeneidade social existente, indicando a personalização do índice de vulnerabilidade de acordo com os cenários e níveis de desenvolvimento. Cardona (2003) agrega a esse pensamento o fato que as causas subjacentes da vulnerabilidade estão ligadas a processos econômicos, demográficos e políticos que afetam a distribuição de renda e entre diferentes grupos de pessoas, e que alguns processos globais afetam a população e exigem mais atenção como: crescimento populacional urbano, insegurança econômica, degradação ambiental e mudanças climáticas.

Aderindo à hipótese de que a falta de desenvolvimento e vulnerabilidade estão correlacionadas, Cardona (2003) sugere que a vulnerabilidade se origina em:

a) fragilidade ou exposição física: a susceptibilidade de um grupo ou comunidade ser afetada por um fenômeno climático, devido à sua localização na área de influência do fenômeno e à ausência de resistência física;

b) fragilidade socioeconômica: a predisposição a sofrer danos pelos níveis de marginalidade e segregação social dos assentamentos humanos, e as condições desfavoráveis e fraquezas relativas relacionadas a fatores sociais e econômicos; e

c) falta ou ausência de resiliência: expressa por limitações de acesso e mobilização dos recursos do assentamento humano e sua incapacidade de responder quando se trata de absorver.

Assim, objetivo desta pesquisa é sugerir um índice de vulnerabilidade populacional aos impactos das secas (IVPopS), em escala de microrregião, uma vez que a cobertura e a disponibilidade de dados climáticos não são satisfatórias no que concerne a escala municipal, quando 
aliado a indicadores demográficos. Desta forma o estudo propõe identificar áreas onde as populações são mais vulneráveis a eventos de seca. Na próxima seção apresenta-se a metodologia de criação do indicador de vulnerabilidade social e na seção seguinte apresenta-se os resultados de um estudo-piloto, e por fim as conclusões.

\section{Material e Métodos}

O ponto de partida deste estudo é a possível criação do IVPopS como uma alternativa aos vários indicadores sociais de vulnerabilidade climática, como o proposto por Hummel et al. (2016). Já está bem estabelecido que as muitas diferenças socioeconômicas, demográficas e de desenvolvimento refletem diretamente uma vulnerabilidade social. Desta forma, analisar a interação climática sobre determinada localidade apresenta uma contribuição significativa à sociedade.

As bases de dados para a ilustração do IVPopS foram obtidas no censo agropecuário de 2006, fornecido pelo Instituto Brasileiro de Geografia e Estatística (IBGE), além de dados de precipitação mensal obtidos da Agência Nacional das Águas (ANA).

Partindo do conceito apresentado pelo AR5 (IPCC, 2014), que sugere que a vulnerabilidade reflete o conjunto de perigos - define-se o evento físico potencialmente prejudicial que uma comunidade está exposta, mitigada pela sua capacidade adaptativa - sua capacidade de responder de forma eficaz ao risco. Desta forma uma expressão que pode ser inicialmente sugerida:

$$
V=f(R, E, S, \overline{A C})
$$

onde $R$ é o fator de Risco (perigo $H$ (de hazard), e exposição $E$ (de exposure)), $S$ é sensibilidade ou fragilidade e $\overline{A C}$ é a incapacidade de adaptação, que é o evento probabilístico complementar à capacidade de adaptação.

$\mathrm{Na}$ realidade a Eq. (1) pode ser de difícil implementação, principalmente em países em desenvolvimento que possui regiões áridas ou semiáridas, por falta de dados que representam a heterogeneidades locais, principalmente, nos aspectos climáticos e socioeconômicos (Simelton et al., 2009). Fato que pode ser comprovando no NEB, faltam dados ou periodicidade das informações, principalmente de fatores biofísicos de alguns grupos sociais, como por exemplo, agricultores que praticam a agricultura familiar.

O perigo $(H)$ é definido como a ocorrência potencial de um evento físico ou tendência do impacto físico natural ou induzido pelo homem - dito de natureza antropogênica - que pode causar perda de vidas, propriedades, infraestrutura socioeconômica, ecossistemas, recursos naturais (IPCC, 2014), e pode ser quantificado pela seguinte equação:

$$
H=P(E) * C
$$

onde $P(E)$ é a probabilidade de um evento extremo ocorrer em determinado local, $C$ é quantidade de pessoas afetados pelo o evento extremo. Desta forma o risco, à um determinado evento extremo é associado a vulnerabilidade V, logo risco (ou exposição à seca), pode ser quantificado pela seguinte equação:

$$
H_{\text {drought }}=f(S P I, D) \Rightarrow f([\exp (D M) * \log (D)]
$$

em que o SPI (McKee et al., 1993), é o índice padronizado de seca que foi calculado usando dados mensais de precipitação disponíveis pela ANA, com faixa temporal de 1 de janeiro de 1979 até 31 de dezembro de 2011. A magnitude de seca (DM - sigla do inglês, Drought Magnitude) e $D$ é a média de desastres de seca por microrregião. A $D M$ foi determinada no período chuvoso, e segue o equacionamento proposto por Mckee et al. (1993):

$$
D M=-\sum_{j=1}^{n} S P I_{i j}
$$

em que $j$ é o mês de início do período chuvoso que vai até o mês $n$, e $i$ representa o ano da série temporal. Adicionalmente, $D$ é o número médio por microrregião de desastres de seca decretados no período de 1991 a 2012, publicado pelo Centro de Estudos e Pesquisas em Engenharias e Defesa Civil, no site do CEPED. E $E$ é o grau de exposição de uma comunidade em termos de população ao evento extremo. Por fim a IVPopS é expressa pela seguinte função:

$$
I V P o p S=f(R, E, S, \overline{A C})
$$

As diversas metodologias para se conceituar e tornar operacional um índice de vulnerabilidade populacional - a social (Adger, 2003; Adger, 2006; Füssel, 2007), a agrícola (Luers et al., 2003; Barbier et al., 2009; Popova et al., 2014; Silva e Lucio, 2014b), a sanitária Confalonieri, (2007) - usam técnicas de Estatística Multivariada. Neste artigo propõe-se atrelar a Teoria de Probabilidades a essas técnicas Multivariadas para determinação do índice de vulnerabilidade IVPopS, como é descrito a seguir. O modelo conceitual considera a dependência entre eventos que são os compartimentos da definição de vulnerabilidade - i.e., a ocorrência de determinado evento A modifica a probabilidade de a ocorrência do evento $\mathrm{B}$.

\subsection{Teorema da multiplicação de probabilidades}

Seja um conjunto finito de eventos $A_{1} \cap A_{2} \cap \ldots \cap A_{n}$ tais que os eventos condicionais $A_{i} \mid A_{1} \cap A_{2} \cap \ldots \cap A_{i-1}$ tenham probabilidades positivas. Então tem-se que: 


$$
\begin{array}{r}
P\left(\bigcap_{i=1}^{n} A_{i}\right)=P\left(A_{i}\right) \cdot P\left(A_{2} \mid A_{1}\right) \cdot P\left(A_{3} \mid A_{1} \cap A_{2}\right) \ldots \\
P\left(A_{n} \mid A_{1} \cap A_{2} \cap \ldots \cap A_{n-1}\right)
\end{array}
$$

Consideramos que o IVPopS seja um uma função de probabilidade descrita da seguinte forma:

$$
\begin{aligned}
& P(R \cap S \cap \overline{A C})=P(H \cap E \cap S \cap \overline{A C})= \\
& P(H) \cdot P(E \mid H) \cdot P(E \mid H \cap E) \cdot P(\overline{A C} \mid H \cap E \cap S)
\end{aligned}
$$

Na Eq. (7), $R$ é o risco (interação entre perigo e exposição), $H$ é o perigo climático, o $E$ é o índice de exposição, o $S$ é o índice de fragilidade populacional e o $\overline{A C}$ é a incapacidade de adaptação. Para a determinação de cada compartimento faz-se uma composição ou combinação (linear ou não linear) de variáveis, criando-se índices.

No que tange ao perigo $H$, pode-se considerar o $S P I$ derivado das precipitações mensais, enquanto para os compartimentos $E, S, \overline{A C}$ pode-se considerar variáveis demográficas, socioambientais, agropecuárias, sanitárias, econômicas entre outras, dependendo do objetivo do estudo. Na composição de cada compartimento utiliza-se técnicas estatísticas multivariadas como análise de componentes principais (ACP) ou análise fatorial (AF).

Para representar os métodos de obtenção de cada componente na função determinada pela Eq. (7), utilizouse a técnica multivariada Análise Fatorial (AF) proposta por (Hair et al., 2010). O principal objetivo da AF é reduzir a quantidade de variáveis através da criação de índices. Estes índices iram gerar variáveis latentes ou fatores que captam a máxima variabilidade do conjunto original de variáveis.

A formulação matemática será apresentada no Apêndice 1 e a tabela com as 33 variáveis que compõem estes fatores será apresentada no Apêndice 2.

\subsection{Aplicações ao NEB}

Através dos dados mensais de precipitação determinou-se climatologicamente a quadra chuvosa no NEB compreende os meses de janeiro a abril. Em seguida aplicou-se a Eq. (4) para determinar a magnitude de seca no período chuvoso com o objetivo de avaliar a qualidade desse período chuvoso, para gerir esses recursos hídricos para garantir o abastecimento local e econômico da melhor forma possível.

Diversos pesquisadores diagnosticaram que a variabilidade pluviométrica está associada a incursão de sistemas atmosféricos de diferentes escalas espaço-temporal ao longo do ano (Rao et al., 1997; Chaves e Cavalcanti, 2001; Souza et al., 2005; Oliveira et al., 2014). Diante desta afirmação os autores através do $M D$ calculado para cada estação meteorológica exibida na Fig. 1a, aplicaram a técnica de cluster para separar os grupos mais homo- gêneos de $M D$ e verificar a variabilidade da precipitação em cada grupo.

Observou-se cinco grupos, e foi realizada a análise climatológica de cada um deles (Fig. 1b). Notou-se que, os grupos G4 e G5 são os mais homogêneos quando comparado aos demais grupos, evidenciado a variabilidade da precipitação, com possível causa a atuação da Zona de Convergência Intertropical (ZCIT) que se posiciona mais à sul neste período (Carvalho e Oyama, 2013).

De acordo com Melo et al. (2009), a ZCIT é o principal sistema que atua na quadra chuvosa do norte do Nordeste, com papel fundamental na qualidade do período chuvoso. Notou-se que, mesmo no período chuvoso a região possui um grau mínimo de seca, além disso os grupos como G3 e G5 possuem menor valor da $M D$ quando comparado com os demais, o G4 é o que possui o maior valor da $M D$ regional.

Para a avaliação da qualidade dos grupos da Fig. 1c, utilizou-se o índice de Silhouette (Si) desenvolvido por Rousseeuw (1987), que avalia como uma observação é semelhante a outras observações inseridas em seu cluster, comparando com as observações inseridas em outros clusters. Cada observação tem um Si, e uma média geral permite avaliar o desempenho geral dos clusters. Os valores desse índice variam no intervalo de -1 a 1 . Os valores próximos a 1 indicam que o objeto está no cluster correto. Os valores próximos a -1 indicam que a observação provavelmente foi alocada a um grupo inadequado, e valores próximos a zero indicam que o objeto está próximo ao limite entre dois clusters.

Este índice foi utilizado por Santos et al. (2015) para caracterizar a precipitação na Amazônia, onde os autores avaliaram que 3 grupos eram suficientes para regionalizar a precipitação obtendo um valor de Si de 0,45 . Nessa pesquisa, obteve-se um valor de Si 0,43 para 5 grupos. Para Alvares et al. (2013), no Nordeste predomina dois tipos de clima: o tropical e semiárido. O clima tropical na região classifica-se em Af (sem estação seca), Aw (com inverno seco) e As (com verão seco).

\section{Resultados e Discussões}

Após aplicação da técnica estatística multivariada AF em cada compartimento do indicador IVPopS, exceto no compartimento de risco. As Figs. 2 e 3 apresentam o resultado da AF e a Fig. 4 a espacialização do IVPopS. A necessidade em dar uma resposta a sociedade sobre o impacto causado pelas mudanças climáticas tem exigido dos pesquisadores um grande esforço em diagnosticar essas áreas de risco, além de implementar ações de planejamento e gestão com a relação do homem com seu ambiente natural (Marandola e Hogan, 2005).

Observa-se na Fig. 2a que a componente de risco descrita na Eq. (2) apresenta baixo risco da faixa do litoral e extremo sul da Bahia e se prolonga por toda faixa do 

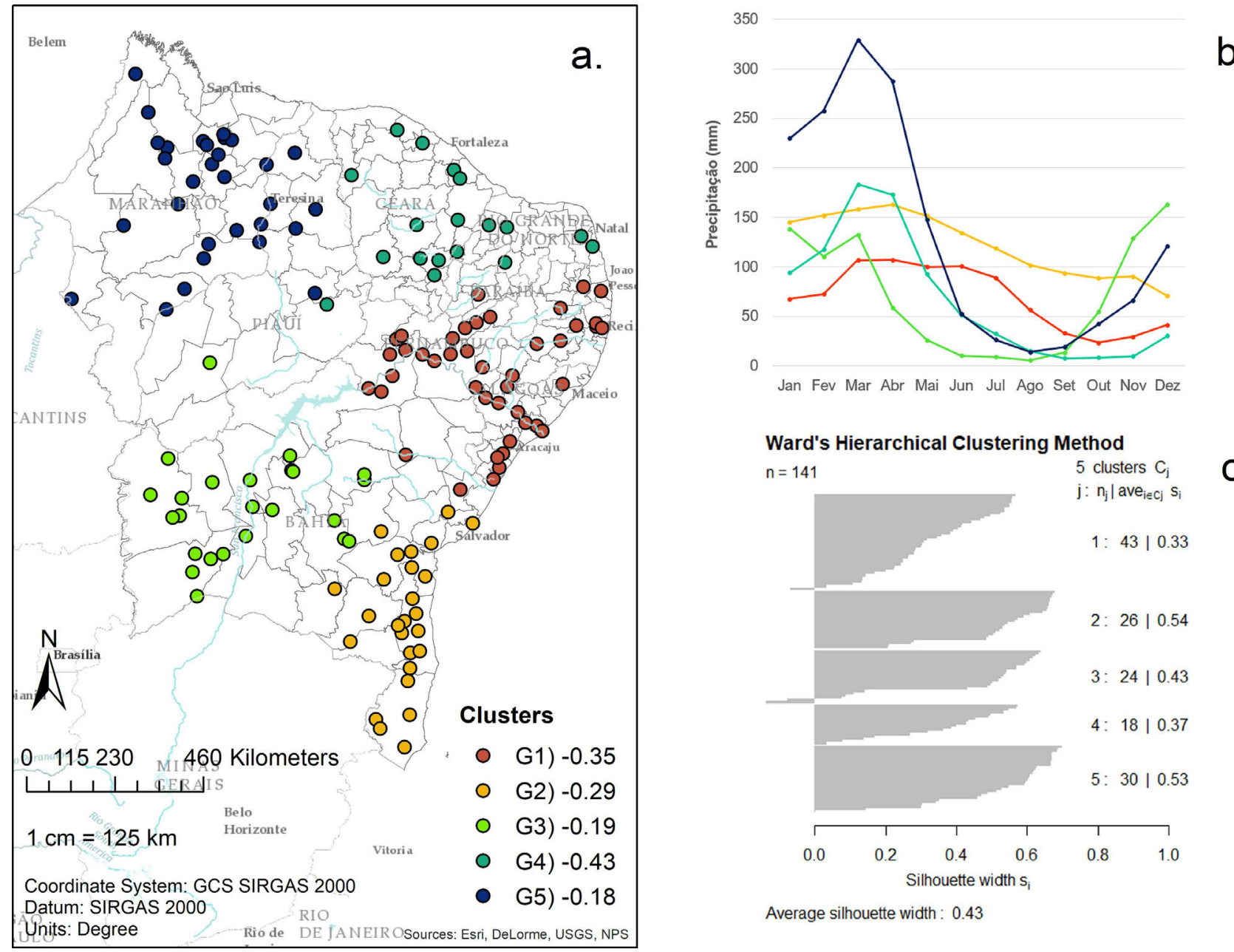

b.

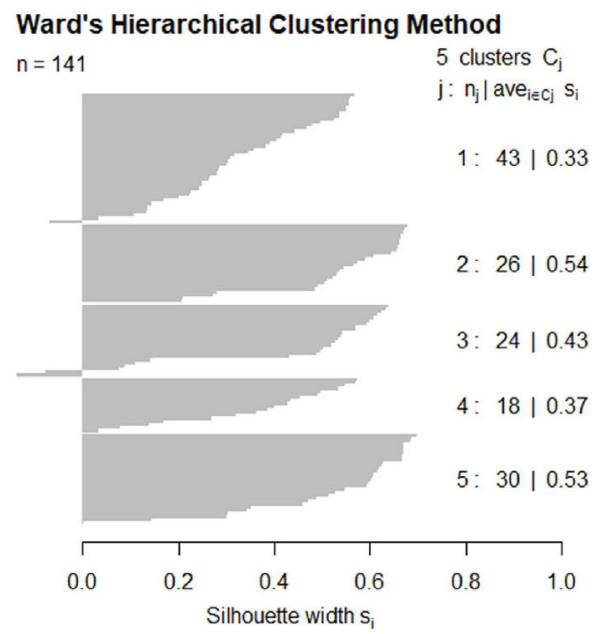

Average silhouette width : 0.43

Figura 1 - Área de estudo destacando a divisão política da região em microrregiões junto com a análise de cluster das estações de precipitação da Agência Nacional das Águas (ANA), utilizando o $M D$ (a). Climatologia da precipitação de cada cluster (b), e validação da análise de cluster via índice Slilhouette (Si) em (c).

litoral leste até o estado de Pernambuco, além do extremo oeste compreendendo parte do estado do Maranhão. Por outro lado, toda faixa que compreende o semiárido nordestino apresenta uma condição de médio a alto risco de seca no período chuvoso (janeiro/abril) do norte do NEB. Principalmente nos estados de Pernambuco, Paraíba, Ceará, Rio Grande do Norte e Piauí. No extremo oeste baiano aparece um núcleo considerado de alto risco, provavelmente associado ao término do período chuvoso do oeste do Nordeste que se dá entre outubro/novembro até fevereiro/março.

$\mathrm{Na}$ Fig. $2 \mathrm{~b}$ a componente de $E$ apresenta poucas áreas consideradas de baixo exposição ao evento extremo, destacando as áreas entres os estados do Maranhão e Piauí, além de áreas no oeste e litoral do estado da Bahia. Estas áreas citadas, exceto o litoral, são conhecidas na literatura pela expressão MATOPIBA, resultante de um a acrônimo formado com as inicias dos estados do Maranhão, Tocantins, Piauí e Bahia. Ela atribui uma extensão geográfica que recorda parcialmente os territórios dos quatro estados citados.

Nos últimos 10 anos diversas transformações socioeconômicas ocorreram nessa região ligadas à ampliação da infraestrutura viária, logística e energética, tendo, entre outras consequências, o surgimento de polos de expansão da fronteira agrícola baseados na adoção de tecnologias agropecuárias de alta produtividade e os seus três produtos principais - soja, milho e algodão - no mínimo dobraram sua produção nesse período (EMBRAPA, 2020).

Desta forma a Fig. 2b, apresenta um resultado coeso, que além de refletir o fator $E$, principalmente por lavouras temporárias e culturas de sequeiro (por exemplo, o algodão herbáceo) destacando os núcleos de alta exposição oeste dos estados do Maranhão e Bahia. Outro fator que boa parte da área de MATOPIBA é inserida no bioma Cerrado, cerca de $60 \%$ do bioma cerrado encontra-se nos limites definidos para a Amazônia Legal, ficando de fora 


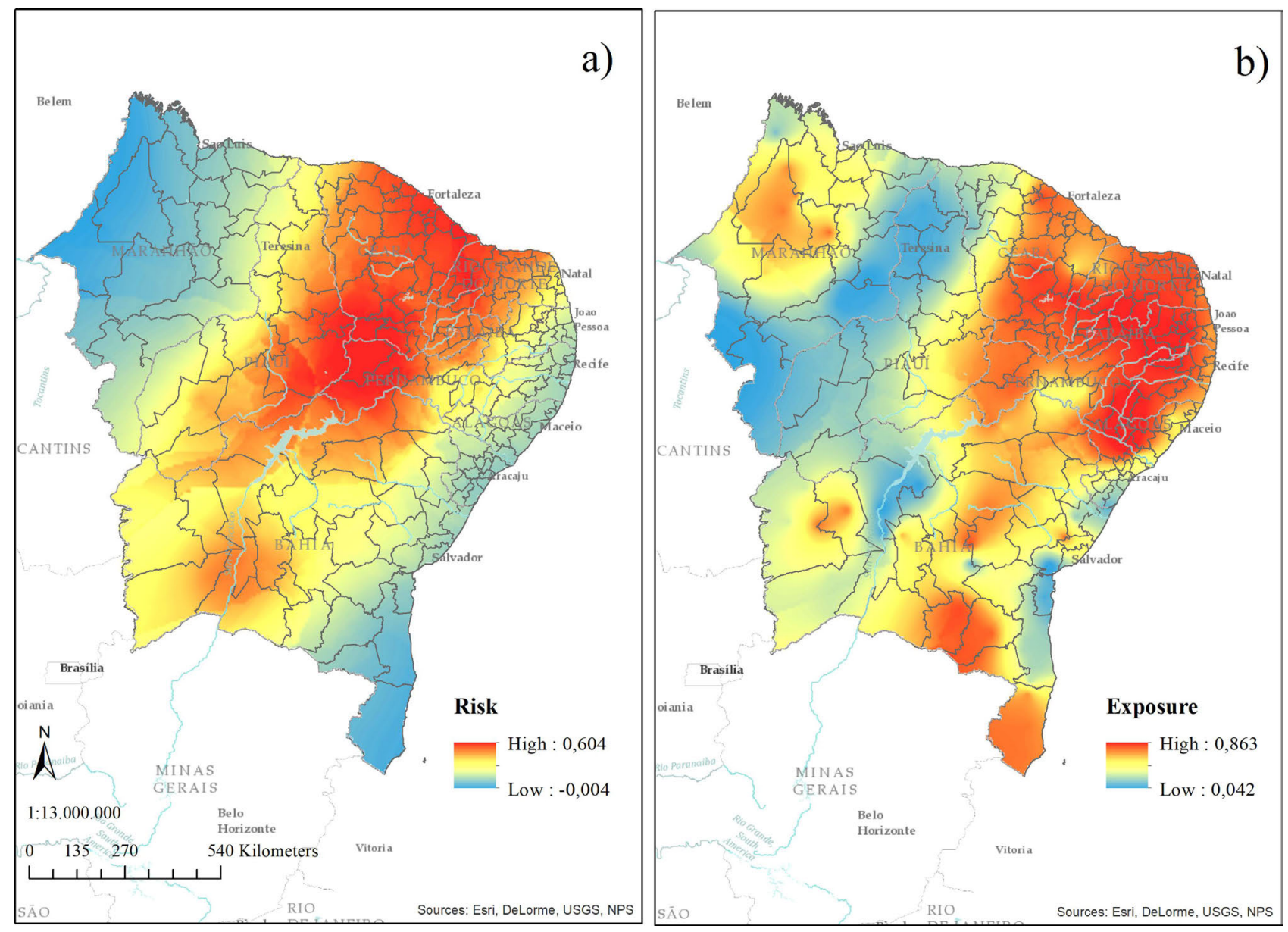

Figura 2 - Espacialização da componente de risco de seca no período chuvoso NEB a) e a componente de exposição ao evento natural b).

os cerrados da Bahia, Piauí e de parte do Maranhão (Brasil, 2012; IBGE, 2014).

A agricultura desempenha um papel fundamental na economia regional, onde $86,2 \%$ a mão de obra empregada no campo é da agricultura familiar (Castro, 2012). Silva e Lucio (2014b) identificaram áreas susceptíveis a eventos extremos de seca, e constatou que as microrregiões do Alto Oeste e Seridó no Rio Grande do Norte, apresentavam os maiores valores do indicador de susceptibilidade corroborando com este resultado.

Na Fig. 3 são mostradas as componentes sensibilidade agrícola (Fig. 3a) e incapacidade populacional de lidar com o evento extremo de seca (Fig. 3b). De acordo com o IPCC (2014), a atividade agrícola nos países em desenvolvimento sentirá mais os efeitos danosos das alterações climáticas, eventos como secas aumentarão de intensidade, assim como a frequência de ocorrência. As áreas que apresentam alta condição de sensibilidade e maior proporção está no estado da Bahia, avançando na direção norte afetando os estados do Pernambuco, Ceará, Piauí e toda costa leste entre Salvador e Bahia. Além de uma área noroeste do estado localizada no Maranhão, por outro lado, o estado do Rio Grande do Norte apresenta valores baixos da componente de sensibilidade agrícola, isso pode estar ligado fatores da dinâmica populacional e uso do solo nestas últimas década, puxado pelo aumento das atividades agropecuárias em cidades, como PetrolinaPE e seu entorno, ou pelo êxodo rural (Rufino e Silva, 2017).

Na Fig. 3b, a incapacidade populacional de lidar com eventos extremos de seca apresenta uma área mais homogênea no norte do NEB, principalmente entre Rio Grande do Norte e Ceará, avançando na direção no estado da Bahia. Observa-se ainda que, mesmo com baixa sensibilidade agrícola, caso a área passe por um evento extremo a incapacidade de superar tal evento é muito alta, como pode ser visto no estado do Rio Grande do Norte.

Justificado pela longa estiagem que passou nestes últimos 10 anos, de acordo com Figueiredo et al. (2016), aproximadamente $93 \%$ da área do estado está na porção semiárida, ou seja, com regimes pluviométricos abaixo de $700 \mathrm{~mm} / \mathrm{ano}$. Aliado falta de investimentos em tecnologias de plantio e uso do solo, baixos índices socioeconômicos evidenciam esta característica de $\overline{A C}$.

Na Fig. 4 têm-se a caracterização espacial do indicador de vulnerabilidade IVPopS, observa-se que a região 

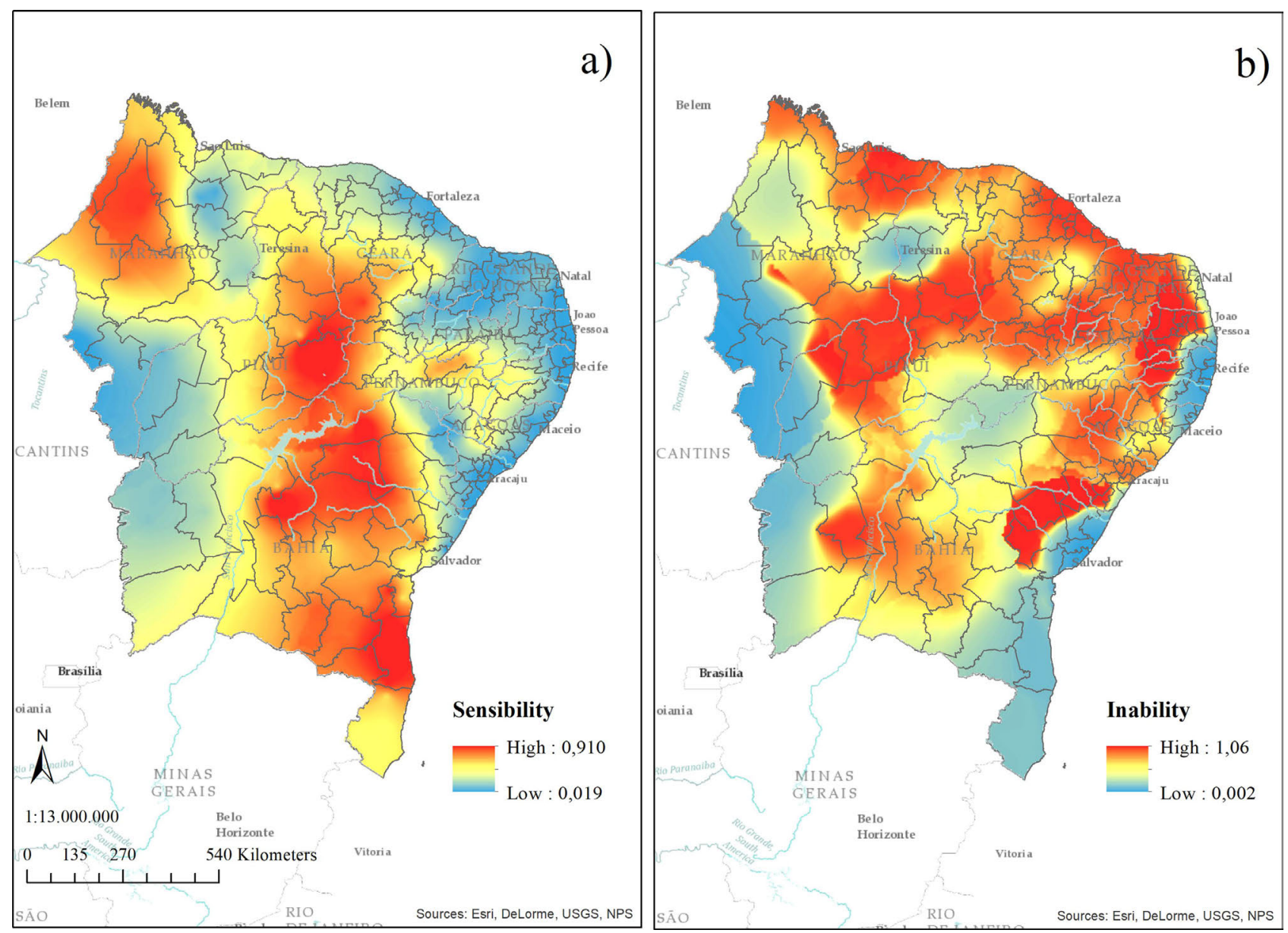

Figura 3 - Espacialização da componente sensibilidade agrícola a) e incapacidade populacional em lidar com evento extremo climático b).

semiárida do NEB apresenta um padrão médio para altos valores do IVPopS, destacando-se o núcleo intersecionando os estados do Ceará, Pernambuco, Piauí e Bahia com alto valor do indicador. Embora os índices de vulnerabilidade, IVPopS e SCVI, sejam diferentes na faixa leste do NEB, sobre a região semiárida os valores o padrão semelhante aos obtidos por (Torres et al., 2012).

\section{Conclusões}

Apresentar uma opção viável e eficaz para a análise de vulnerabilidade no Nordeste, considerando a união de diversos bancos de dados e apresentar uma metodologia estocástica e probabilística tornam o IVPopS representativo ao incorporar uma complexidade inerente as próprias variáveis que o compõem, além de apresentar resultados satisfatórios quando comparados com a literatura citada.

A caracterização do fator de risco seguindo a metodologia descrita nas Eqs. (2) e (3) de acordo com estes valores de probabilidades $(0,004-0,604)$. Segundo ele, a área que apresenta maior perigo abrange os estados do Ceará, Rio Grande do Norte, Paraíba, Pernambuco e Piauí. Por outro lado, o extremo oeste do NEB e o extremo sul, além da faixa da costa leste apresentam os menores valores de perigo de seca no NEB. De acordo com a incapacidade populacional de lidar com eventos extremos de seca, as atividades econômicas dos agricultores familiares no NEB mostram maior exposição, pois há eventos de seca na faixa de 0,042-0,863. Essa exposição foi calculada pela técnica multivariada $\mathrm{AF}$, que apresentou os três componentes que representam as atividades dessas famílias: gado de corte, gado leiteiro e culturas temporárias.

De acordo com o fator de sensibilidade agrícola e incapacidade de adaptação, o NEB possui uma sensibilidade agrícola na faixe de $(0,019-0,910)$ com núcleos expressivos de alta sensibilidade agrícola. Finalmente, a faixa mais oriental que vai do estado da Bahia até o litoral do Piauí tem baixa sensibilidade. Quanto a incapacidade de se adaptar, o NEB ficou bastante evidentes os núcleos de maior IVPopS nos estados do Rio Grande do Norte, Paraíba, Pernambuco, Bahia e pequenas áreas no Maranhão.

Embora existam áreas do semiárido nordestino com pouca sensibilidade a eventos extremos de seca, atividades como a agricultura familiar são incapazes de absorver e lidar com tais eventos. 


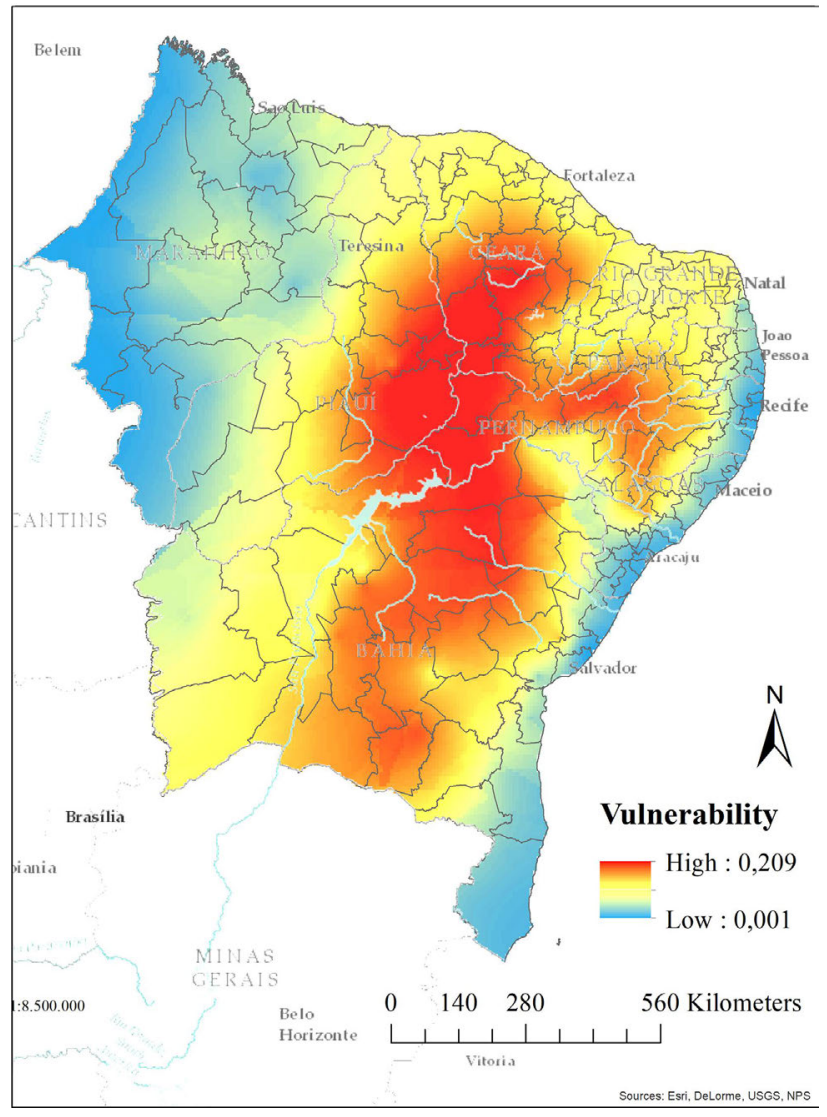

Figura 4 - Espacialização do modelo conceitual de vulnerabilidade populacional IVPopS para o NEB.

\section{Material Suplementar}

Apêndice 1 - Análise Fatorial.

Apêndice 2 - Tabela de variáveis utilizadas na AF para estimação do IVPopS.

\section{Referências}

ADGER, W.N. Social capital, collective action, and adaptation to climate change. Economic Geography, v. 79, n. 4, p. 387 404, 2003.

ADGER, W.N. Vulnerability. Global Environmental Change, v. 16, n. 3, p. 268-281, 2006.

ALVARES, C.A.; STAPE, J.L.; SENTELHAS, P.C.; DE MORAES GONÇALVES, J.L.; SPAVORECK, G. Köppen's climate classification map for Brazil. Meteorologische Zeitschrift, v. 22, n. 6, p. 711-728, 2013.

BARBIER, B.; YACOUBA, H.; KARAMBIRI, H.; ZOROMÉ, M.; SOMÉ, B. Human vulnerability to climate variability in the Sahel: Farmers' adaptation strategies in Northern Burkina Faso. Environmental Management, v. 43, n. 27, p. 790-803, 2009.

BRASIL, Ministério da Casa Civil. Lei n. 12.651, de 25 de maio de 2012. Disponível em http://www.planalto.gov.br/ccivil_03/_ato2011-2014/2012/lei/112651.htm, acesso em 19 de agosto de 2020.
BROOKS, N.; ADGER, N.W.; MICK, K.P. The determinants of vulnerability and adaptive capacity at the national level and the implications for adaptation. Global Environmental Change, v. 15, n. 2, p. 151-163, 2005.

CARDONA, O.D. The Need for rethinking the concepts of vulnerability and risk from a holistic perspective: A necessary review and criticism for effective risk management. In: Mapping vulnerability: Disasters, Development and People. London: Earthscan Publishers, 2003.

CARVALHO, M.A.V.; OYAMA, M.D. Variabilidade da largura e intensidade da Zona de Convergência Intertropical atlântica: Aspectos observacionais. Revista Brasileira de Meteorologia, v. 28, n. 3, p. 305-316, 2013.

CASTRO, C.N. A Agricultura no Nordeste Brasileiro: Oportunidades e Limitações ao Desenvolvimento. Rio de Janeiro: IPEA, 48p, 2012.

CHAVES, R.R.; CAVALCANTI, I.F.A. Atmospheric circulation features associated with rainfall variability over Southern Northeast Brazil. Monthly Weather Review, v. 129, n. 10, p. 2614-2626, 2001.

CONFALONIERI, U.E.C. Mudança climática global e saúde: Perspectivas para o Brasil. Revista Multiciência, n. 8, p. 48-64, 2007.

EMBRAPA, Empresa Brasileira de Pesquisa Agropecuária. Sobre o Matopiba. Disponível em https://www.embrapa. br/tema-matopiba/sobre-o-tema, acesso 20 de agosto de 2020.

FIALHO, E.S.; SOUZA, J.J.L.L. Utilização do clima no planejamento como reflexo das condições ambientais nos centros urbanos. Revista Acta Geográfica, v. 1, n. 2, p. 29-41, 2007.

FIGUEIREDO, S.C.S.; SARAIVA J.J.C.; FIGUEIREDO, J.S. Política de combate dos efeitos da seca no semiárido potiguar: O caso de Riacho do Sangue, em Macaíba/RN, 20022010. Caderno de Geografia, v. 26, n. 45, p. 201-223, 2016.

FÜSSEL, H-M. Vulnerability: A generally applicable conceptual framework for climate change research. Global Environmental Change, v. 17, n. 2, p. 155-167, 2007.

FRANCHITO, S.H.; RAO, V.B.; SANTO, C.M.E; GAN, M.A. An update on the rainfall characteristics of Brazil: Seasonal variations and trends in 1979-2011. International Journal of Climatology, v. 36, n. 1, p. 292-302, 2015.

GUIQIN, J.; FULIANG, Y.; YONG, Z. An analysis of vulnerability to agricultural drought in China using the expand grey relation analysis method. Procedia Engineering, v. 28, p. 670-676, 2012.

HAINES, A.; KOVATS, R.S.; CAMPBELL-LENDRUMB, D.; CORVALANB, C. Climate change and human health: Impacts, vulnerability and public health. Public Health, v. 120, n. 7, p. 585-596, 2006.

HAIR, J.F.; BLACK, W.C.; BABIN, B.J.; ANDERSON, R.E. Multivariate Data Analysis, 7th ed. New Jersey: Prentice Hall, 2010.

HUMMELL, B.M.L; CUTTER, S.L.; EMRICH, C.T. Social vulnerability to natural hazards in Brazil. International Journal of Disaster Risk Science, v. 7, n. 2, p. 111-122, 2016.

IBGE. Vegetação - Cobertura Atual. Disponível em https:// atlasescolar.ibge.gov.br/images/atlas/mapas_brasil/brasil_vegetacao.pdf, acesso em 19 de agosto de 2020. 
IPCC. Summary for policymakers. In: DOKKEN, D.J. et al. (Org.) Climate Change 2014: Impacts, Adaption, and Vulnerability. Part A: Global and Sectoral Aspects. Cambridge: Cambrige Univesty Press, p. 1-32. 2014. Disponível em http://ipcc-wg2.gov/AR5/images/uploads/ WG2AR5_SPM_FINAL.pdf.

KIENBERGER, S.; LANG, S.; ZEIL, P. Spatial vulnerability units - Expert-based spatial modelling of socio-economic vulnerability in the Salzach catchment, Austria. Natural Hazards and Earth System Science, v. 9, n. 3, p. 767-778, 2009.

LUERS, A.; LOBELL, D.B.; SLAR, L.; ADDAMS, C.L.; MATSON, P.A. A method for quantifying vulnerability, applied to the agricultural system of the Yaqui Valley, Mexico. Global Environmental Change, v. 13, n. 4, p. 255-267, 2003.

MARANDOLA JR., E.; HOGAN, D.J. Vulnerabilidades e riscos: entre geografia e demografia. Revista Brasileira de Estudos de População, v. 22, n. 1, p. 29-53, 2005.

MCKEE, T.B.; DOESKEN, N.J.; KLEIST, J. The relationship of drought frequency and duration to time scales. In: Proceedings of the 8th Conference on Applied Climatology, v. 17, p. 179-183, 1993.

MELO, A.B.C.; CAVALCANTI, I.F.A.; SOUZA, P.P. Zona de Convergência intertropical do Atlântico. In: Tempo e Clima no Brasil. São Paulo: Oficina de Texto, 2009.

NÓBREGA, R.S.; SANTIAGO, G.A.C.F.; SOARES, D.B. Tendências do controle climático oceânico sob a variabilidade temporal da precipitação no Nordeste do Brasil. Revista Brasileira de Climatologia, v. 18, p. 276-292, 2016.

OBERMAIER, M.; ROSA, L.P. Mudança climática e adaptação no Brasil: Uma análise crítica. Estudos Avançados, v. 27, n. 78, p. 153-176, 2013.

OLIVEIRA, P.T.; SANTOS E SILVA, C.M.; LIMA, K.C. Linear trend of occurrence and intensity of heavy rainfall events on Northeast Brazil. Atmospheric Science Letters, v. 15, n. 3, p. 172-177, 2014.

POPOVA, Z.; IVANOVA, M.; MARTINS, D.; PEREIRA, L.S.; DONEVA, K.; ALEXANDROV, V.; KERCHEVA, M. Vulnerability of Bulgarian agriculture to drought and climate variability with focus on rainfed maize systems. Natural Hazards, v. 74, p. 865-886, 2014.
RAO, V.B.; SÁ, L.D.A.; FRANCHITO, S.H.; HADA, K. Interannual variations of rainfall and corn yields in Northeast Brazil. Agricultural and Forest Meteorology, v. 85, n. 12, p. 63-74, 1997.

ROUSSEEUW P.J. Silhouettes: A graphical aid to the interpretation and validation of cluster analysis. Journal of Computational and Applied Mathematics, v. 20, p. 53-65, 1987.

RUFINO, I.A.A.; SILVA, S.T. Análise das relações entre dinâmica populacional, clima e vetores de mudança no semiárido brasileiro: Uma abordagem metodológica. Boletim de Ciências Geodésicas, v. 23, n. 1, p. 166-181, 2017.

SANTOS, E.B.; LUCIO, P.S.; SILVA, C.M.S. Precipitation regionalization of the Brazilian Amazon. Atmospheric Science Letters, v. 16, n. 3, p. 185-192, 2015.

SIMELTON, E.; FRASER, E.D.G.; TERMANSEN, M.; FOSTER, P.M.; DOUGILL, A.J. Typologies of crop-drought vulnerability: An empirical analysis of the socio-economic factors that influence the sensitivity and resilience to drought of tree major food crops in China (1961-2001). Environmental science \& Policy, v. 12, n. 4, p. 438-452, 2009.

SILVA, B.K.N.; LUCIO, P.S. Characterization of risk/exposure to climate extremes for the Brazilian Northeast-case study: Rio Grande do Norte. Theoretical and Applied Climatology, v. 122, p. 59-67, 2014a.

SILVA, B.K.N.; LUCIO, P.S. Indicator of agriculture vulnerability to climatic extremes. A conceptual model with case study for the Northeast Brazil. Atmospheric and Climate Sciences, v. 4, n 2, p. 334-345, 2014 b.

SOUZA, E.B.; KAYANO, M.T.; AMBRIZZI, T. Intraseasonal and submonthly variability over the Eastern Amazon and Northeast Brazil during the autumn rainy season. Theoretical and Applied Climatology, v. 81, p. 177-191, 2005.

TORRES, L.L.; LAPOLA, D.M.; MARENGO, J.A.; LOMBARDO, M.A. Socio-climatic hotspots in Brazil. Climatic Change, v. 115, p. 597-609, 2012.

Centro de Estudos e Pesquisas em Engenharias e Defesa Civil (CEPED) http://www.ceped.ufsc.br/.

License information: This is an open-access article distributed under the terms of the Creative Commons Attribution License (type CC-BY), which permits unrestricted use, distribution and reproduction in any medium, provided the original article is properly cited. 